\title{
Living Outside Protocol: Polyamorous Orientations, Bodies, and Queer Temporalities
}

\section{Charlotta Carlström $^{1}$ D $\cdot$ Catrine Andersson ${ }^{1}$}

Published online: 15 June 2019

(c) The Author(s) 2019

\begin{abstract}
This study investigates experiences of polyamory in a society where monogamy is the norm. Polyamory is when more than two people are involved in an intimate and/ or sexual relationship. The relationships are known to those involved, and everyone has the opportunity to have multiple relationships at the same time. In-depth interviews were completed with 22 persons in Sweden who identify as polyamorous. Drawing on Ahmed's phenomenological concepts of turning points and lines and Halberstam's concept of queer time and temporality, the following questions are explored: What turning points can be seen in the informants' stories? And what consequences are the informants exposed to when heteronormative expectations are not followed? In the theoretical language of Ahmed, living a life within monogamous boundaries would be considered as being "in line". Going beyond these monogamous heteronormative lines can result in more relational choices by which one has to find out what kind of relationship works best instead of following a ready-made template. The majority of the informants feel forced to conceal their relationship constellations in several situations and contexts. Living a queer life is seen by others in society as not only incomprehensible but also immature and inexperienced. Interactions with healthcare professionals seldom offer any relief from this; instead, the informants' stories of these encounters can be interpreted as instances of being stopped and blocked, resulting in stress and shame.
\end{abstract}

Keywords Polyamory $\cdot$ Sweden $\cdot$ Turning points $\cdot$ Lines $\cdot$ Queer time $\cdot$ Temporality

\section{Introduction}

The concept of 'non-monogamy' aims to reflect a diversity of alternative relationship constellations that exist beyond the heteronormative. Non-monogamy can be regarded as an umbrella term for polyamory, open relationships, swingers, and

Charlotta Carlström

charlotta.carlstrom@mau.se

1 Department for Social Work, Malmö University, Malmö, Sweden 
relationship anarchy to mention some of the most common. Polyamory, which we focus on here, means that more than two people are involved in an intimate and/ or sexual relationship. The relationships are known to those involved, and everyone has the opportunity to have multiple relationships at the same time. Polyamory has received growing attention in recent years, and there is evidence that an increasing number of people are forming polyamorous relationships (Barker 2005; Haupert et al. 2017). Increasing media attention about polyamory and psychotherapeutic 'self-help' style books accompany this. Non-monogamy has also received increased attention within academia.

In this article, we explore the lines that lead to experiences of polyamory in a society where monogamy is the norm. The marginalization of non-monogamous practices in heteronormative and mononormative contexts is explored by focusing on aspects such as the concept of mononormativity (Barker 2005; Ritchie and Barker 2006), stigma (Conley et al. 2013; Hutzler et al. 2016) or discrimination and privilege (Klesse 2018; Palotta-Chiarolli 2010). In our analysis, we could see that our material supports these previous studies in terms of how the interviewees talked about silence and negative reactions, but there was also a strong narrative about the possibilities that living outside of protocol offered the interviewees. These possibilities were often described in temporal and embodied terms in relation to other polyamorous or queer bodies that seemed to "open up" forays into the previously unthinkable. Therefore, we wanted to explore how non-monogamy can be seen as a work of stepping outside the trodden path with others and imagining these relationships in new temporal light. Drawing on Sara Ahmed's (2006) phenomenological concepts of turning points and lines and Jack Halberstam's (2005) concept of queer time and temporality, the questions investigated are: What turning points can be seen in the informants' stories? And what consequences are the informants exposed to when heteronormative expectations are not followed? Research on polyamorous relations can generate an understanding of contemporary meanings and practices of forming relations as well as of the needs of individuals and families with intersecting marginalized identities. In our study, we aim at understanding the experiences and consequences of living a non-monogamous life, and more specifically, how these experiences are shaped in relationship to other polyamorous and queer bodies.

In the period from the late-1990s until the mid-2000s, special issues on nonmonogamy appeared in several journals, and Barker and Langdridge's (2010) edited anthology Understanding Non-monogamies (2010) contributed to make polyamory accessible to a wider readership. The body of empirical research on polyamory has been growing since the mid-2000s, and according to Klesse (2018), the different studies show the range of multiple locations that emerge from polyamorous identities, practices, and communities. According to Haupert et al. (2017), every fifth person in the United States has experienced a consenting non-monogamous relationship. More men than women reported previous participation in a consenting non-monogamous relationship, as did people who identified as gay, lesbian, or bisexual compared to those who identified as heterosexual. The people who reported having engaged in a non-monogamous relationship varied by gender and sexual orientation but did not differ by age, education level, income, religion, geographic region, political affiliation, or race (Haupert et al. 
2017). Balzarini et al. (2018) examined demographic differences between 2428 individuals in polyamorous relationships and 539 individuals in monogamous relationships in the United States. The result shows that those in polyamorous relationships were more likely to report minority sexual identities, be in a civil union, be divorced, and earn less than $\$ 40,000$ per year compared to individuals in monogamous relationships.

Furthermore, previous studies have focused on various areas related to polyamory such as sexual orientation (Tweedy 2011; Haupert et al. 2017), agreements and trust (Wosick-Correa 2010), jealousy (Deri 2015), emotions and intimacy (Klesse 2018), and attitudes towards polyamory and non-monogamy (Séguin 2017). Overlaps between polyamory and BDSM have also been noted in previous research (Carlström and Andersson 2019; Bauer 2010), and some studies have also shown a relationship between bisexuality and polyamory (Klesse 2006; Kolesar 2011; Sheff 2005). In the Kolesar (2011) study, approximately half of the polyamorous participants identified as bisexual. In Sweden, the limited research available on polyamory has mainly come from studies on bisexuality (Gustavson 2006; BertilsdotterRosqvist 2007).

Several studies investigate attitudes towards consensual non-monogamy (Rubin et al. 2014; Conley et al. 2013; Matsick et al. 2014; Hutzler et al. 2016). Conley et al.'s (2013) research shows that people engaged in non-monogamy are thought to have poorer relationships and be less responsible than people in monogamous relationships. Matsick et al. (2014) investigate differences in attitudes towards subtypes of non-monogamy and demonstrate that swingers were overwhelmingly perceived more negatively and less responsible than individuals in polyamorous relationships. The researchers suggest that people are more uncomfortable with the idea of strictly sexual relationships than relationships involving multiple romantic and/or emotional attachments. In Hutzler et al.'s study (2016), individuals reporting more traditional traits regarding political conservatism and religiosity also reported more negative attitudes towards polyamory.

The life situation of persons practicing polyamory is characterized by invisibility, the risk of discrimination, and exclusion from any family law protection of their relationships (Anapol 2010; Barker and Langdridge 2010; Conley et al. 2013). Pallotta-Chiarolli (2010) details how polyamorous families fear of legal action, social stigma, and harassment, resulting in a majority of the families hiding their polyamorous family structure. Tweedy (2011) describes how the risk of rejection and condemnation from family, friends, and colleagues prevents many non-monogamous people from being open about their life choices. According to Sheff and Hammers (2011), financial and social resources can protect people to a certain extent from some of the risks related to living outside the norm, such as losing employment, custody, or social relationships. Williams and Prior's (2015) study reveals great ignorance of non-monogamous relationships among professionals working in, for example, schools, healthcare, and social services.

Sociological research specifically on queer polyamory is limited, although with some exceptions. Pain (2019) investigates family practices and adaptive strategies among LGBTQ and polyamorists. In addition, Deri (2015) highlights the social and structural context that surrounds jealousy within polyamorous relationships. 
Wilkinson (2010) focuses on polyamory and potential future moves towards political mobilization, and Klesse (2007b) writes about non-monogamous sexual relationships among gay men and bisexuals. Research on polyamory with a queer perspective is important for several reasons; as Pain (2019) notes, many queer voices have been marginalized in literature on polyamory. Studying polyamory with a queer perspective will contribute to the conversation on the development of contemporary lifestyles and can give a clearer picture of what queer polyamory means for LGBTQ politics, particularly in regard to the process of assimilationism (Pain 2019). Our study contributes to the queer literature on polyamorous and queer life choices, as it explores experiences of polyamorous lines and queer temporalities within these relationships. Specifically, we aim to contribute by further exploring how polyamory is practiced in relation to other polyamorous and queer bodies and how, in some contexts, this means sometimes being stopped and in other contexts it means being able to open up and step into the unknown.

\section{Method}

In total, twenty-two persons who identify as polyamorous or non-monogamous were interviewed. Purposeful sampling was applied, and the inclusion criteria were that the interviewees should be 18 years or older with experiences of non-monogamy. The informants were between twenty and sixty years old. Eleven persons from the sample identified as women, three as non-binary, and eight as men. Most were born and raised in Sweden, although a few had another Nordic, central European or southwest Asian background. Two-thirds of the informants live in one of the three largest cities in Sweden, and one-third live in another city or rural area. All the informants had finished high school, and many have a university degree. Most worked or studied, but a few were searching for employment. Our selection included a polyamorous people who were involved in relationships where all partners have the opportunity to have multiple partners, which means that we did not include polygyny or polyandry, which is where only the men or only the women have multiple partners.

We conducted in-depth interviews starting with questions about how the informants defined polyamory and non-monogamy and how they initially got involved with it. The interviews were conducted in the informants' homes, at cafés, and at the university. Each interview, which was conducted in Swedish and translated into English by the authors, lasted between one to three hours. Our study did not set out to prove hypotheses or to test theory, but rather it sought to generate empirical data from which an understanding might be developed. In the analysis work of the interview material, the thematic analysis method as proponed by Braun and Clarke (2006) was applied. The initial analysis showed the importance of themes such as dramatic life changes, encounters with polyamory, encounters with expectations of unreliability, and low awareness of polyamory. These themes often contained temporal and embodied aspects relating to other polyamorous or queer bodies that seemed to "open up" forays into the previously unthinkable. The prominent categories related to these themes were explored by using the theoretical concepts of turning points and lines (Ahmed 2006) and Jack Halberstam's (2005) concept of queer time and 
temporality. We continuously modified the use of the theoretical concepts in a dialectical relationship with the stories of the informants. In the quotations, redundant words like "uhm", "yes", "well" and similar, were removed for greater readability. In the analysis, we used pseudonyms to protect the informants' identities and to guarantee them confidentiality.

Throughout the entire project, ethical considerations played a central role. Informed consent was obtained from all the individual participants included in the study. The project follows the Swedish Research Council's ethical guidelines (Codex 2012), which involves informing the participants of the current research assignment and obtaining consent from the participants, giving them the highest possible confidentiality and assurance that collected data are for research purposes only. The project was reviewed and approved by the Regional Ethical Review Board in Lund, Sweden.

\section{Theory}

\section{Lines and Turning Points}

According to Ahmed (2006), it is through our bodies that we exist in the world and bodies constitute the boundary between an outer and an inner world. She brings the queer theoretical conversation into dialogue with phenomenology with its focus on bodies and spatiality. Ahmed understands bodies to take shape from reiterations and that bodies are always closely linked to sexuality. Partly as a biological body with biochemical and physiological processes, and partly, which is central to phenomenology, as a symbol-bearing and meaning-creating subject. Therefore, Ahmed (2006) is interested in the body's orientation in the world. Orientation is about where we are, where we are going, and how we get there, and she argues that sexuality is about being oriented. Orientation is also about how bodies inhabit and occupy space in the world. Ahmed (2006) uses lines as a theoretical tool to describe "how the body gets directed in some ways more than others" (p. 15), and when some paths or lines are chosen, others are at the same time not chosen. What Ahmed describes as the "straight line" is not visible, which makes it "natural" or the norm: "Being in line allows bodies to extend into spaces that, as it were, have already taken their shape" (p. 15). The heteronormative line goes straight through the cultural system, ensuring that monogamous heterosexuality is maintained as a desirable, obvious, and naturally given way of life. Ahmed uses the path as a metaphor to explain heterosexuality as an orientation. A path is made by the repetition of being trodden upon. Ahmed (2006, p. 16) states, "A paradox of the footprint emerges. Lines are both created by being followed and are followed by being created." The bodies that do not fit in the heteronormative lines end up off course; they lose their orientation. But it is also possible for a body to have a queer orientation despite the hegemonic power directing us in heteronormative lines. New lines and turning points are created when a queer body comes into contact with another queer body, which requires the body to be reoriented. As a result, new objects or bodies, which were previously not visible or achievable within the heteronormative lines, appear to be achievable. 
In other words, a body needs contact with other objects or bodies in order to change orientation to become, for example, lesbian or polyamorous (Ahmed 2006).

We find Ahmed's focus on lines and her description of heterosexuality as "being in line" fruitful in relation to polyamory, as it can contribute to a theoretical understanding of the interviewees' experiences of not following the trodden path of heterosexual togetherness and being oriented toward other queer bodies.

\section{Queer Time and Temporality}

Our existence is neither planned nor coincidental but rather organized on the basis of a heterosexual social order of power-what Jack Halberstam (2005) calls life schedule. The different phases of the life schedule are central to the organization of life and can be defined as birth, children, teens, youth, adults, middle age, old age, and death. For every phase, there are norms and expectations which reflect contemporary ideas of how life should be lived at that particular time. The different phases from childhood to old age can therefore be said to contain different moments of expected maturity. If we act according to our age, it means that we pass the agecoded categories of life correctly and have a better chance of avoiding stigmatization. The life phases are naturalized based on a given heterosexual regulation, which can be explained by the term heteronormativity (Rosenberg 2002). This means that we are born into a social community dominated by heterosexual norms which are also closely connected to social class (Skeggs 1997). Halberstam points out that life stages are surrounded by class-bound rituals, and if we act in accordance with our age, gender, and class, then we pass properly through the fixed categories in life and maintain an expected and age-bound heterosexuality. By doing so, we gain recognition as comprehensible and responsible, and therefore, respectable. Queer time, in contrast to this, is one that "leaves the temporal frames of bourgeois reproduction and family, longevity, risk/safety, and inheritance" (Halberstam 2005, p. 4). It has "the potentiality of a life unscripted by the conventions of family, inheritance, and child rearing" (Halberstam 2005, p. 2). Queer time and temporality thus offer an alternative framework for the theorization of disqualified and anticanonical knowledges of queer practices (Dinshaw et al. 2007).

Taken together, Halberstam and Ahmed provide a foundation for investigating the formation of polyamorous relationships. Both Halberstam's exploration of the phases of life schedule and Ahmed's phenomenological exploration of lines and turning points theorize important aspects of forming relationships in contemporary society. When read together, they provide a queer perspective from which to examine polyamorous experiences together with other queer bodies and the potential of unscripted life in a society where monogamy is the norm. In the next chapter, we analyze the empirical material with the help of these theoretical tools. 


\section{Analysis}

\section{To Re-orient the Body}

All informants tell about their first experiences of non-monogamy. Several of the informants testify to a certain moment in their life, which they refer to as a turning point. Kristian lives with Karin, and they see each other as primary partners and both have love relationships with others. Kristian, who did not have any polyamorous experiences before he met Karin, talks about when they first met as a turning point and the possibilities this had for becoming oriented toward a polyamorous body for the first time:

I thought it was fucking strange [laughing], but I was instantly in love with her... I had just come out of a relationship that had been terrible for two or three years, and I had had a couple of relationships before that which had not worked either. The common denominator was jealousy. Then I thought, "What the hell - it has not worked out in other ways, why not try this and we'll see if it works or not? At worst, I'll learn something anyway." So we tried, and it did work out.

According to Ahmed (2006), "Queer orientations are those that put within reach bodies that have been made unreachable by the lines of conventional genealogy" (p. 107). Jasmine describes how she was approximately thirty when she started exploring non-monogamous relations. "Why at that time?" the interviewer asked, and she answered,

Yes, well, I divorced. It was a pretty horrible marriage, with that woman. It was, yes, it was quite traumatic. Then after that, it became like some kind of release party, for the whole [of] life. And I started exploring everything possible. I had a friend who was in a poly community and all that. They were three people in a relationship, and it seemed so exciting but also that you still can be relaxed in it in some way. And that was where something inside woke up - like this is probably my thing. Because it fits in with all the things that didn't fit in before.

Jasmine's story contains several aspects of how the shift felt-that it fit, like something inside waking up and being able to feel relaxed. As long as Jasmine was in a monogamous marriage, the polyamorous lines were not reachable. When she divorced, her body became oriented towards other forms of relationships than the monogamous, and she began to explore a polyamorous lifestyle and realized that it suited her-it felt like her thing. For Stefan, it was difficult to understand that he had the ability to love more than one person. He says,

It was very difficult at first to realize that I loved another woman. Because I had like this wall; I love Sanna, then there is no one else I can love. It was not something I thought was possible. 
Stefan describes the difficulty he experienced in transgressing the norm of living with one person as a wall-something preventing him from going further in that direction. Ahmed's (2006) lines are closely related to the concept of norms, but they do not have identical meanings. Bremer (2011) describes the line concept as a spatialization of the norm concept. Although norms affect how people orient themselves in life, Ahmed's queer phenomenology enables an analysis focusing on the relationship between bodies and space. In human interactions, there are expectations to both follow and relate to the lines already created by others. Like several other informants, Stefan had incorporated feelings and thoughts of what is appropriate and inappropriate in earlier socialization processes; therefore, the process of becoming polyamorous requires that the persons must shape new values and approaches that are in line with the non-monogamous lines and norms. Stefan continues, "The advantage of coupledom is that it is easy, "cause you know how it should be." Linn also describes how she has dealt with the internationalized norms:

I've broken a lot of the norms telling you how to live, what's right for a woman of my age to do (...) Earlier, I lived my life according to a complete protocol. I had opinions that norms are oppressive and prevent our own personal happiness and freedom. But it was only when I was at the end of my 30s that I realized that "Shit, this applies to me as well." And then I started to deal with things: I realized I didn't want kids. I didn't wanna be monogamous. I wasn't straight. So, I had to abandon the person I thought I was and become the person I really am and actually have been all along. I can get a bit bitter [when I] think, "Did it really have to take such a long time to get here?"

On the one hand, Linn talks about becoming someone that she has been all along, but on the other hand, she also describes it as a path she had to walk to "get here"-one that, in her opinion, perhaps took too long. Descriptions of given templates and norms that act as guidelines in the life of the informants are recurrent in the stories. In Linn's interview, she tells of how both age and gender become important components in the performance of achieving respectability when she describes how she has violated the norms of what is correct behavior for a woman her age (Skeggs 1997). When we realize that our desires do not fall in line with the heterosexual norms in society, then what Ahmed (2006) defines as a "horizontal moment" occurs. It is not a given how we experience the insight that we are about to change course, but rather it can be described as moving in a dark room-to be disoriented (Ahmed 2006). Jonas compares monogamous relations to polyamorous relationships:

The monogamous relationship consists of complete rules and frameworks. People can fall in love, and then they say, "Okay, but now we're together" and they never need to talk about "okay, what do these rules mean and what do you want and need in our relationship?" Because it's like a package you just buy. But as soon as you move outside the monogamous form, you have 
to start defining yourself and your partner or partners and discuss "how do we want to live together?"

Going beyond the monogamous heteronormative frameworks can result in more choices and that one has to find out what kind of relationship works best instead of following a ready-made template. And this is something that Jonas imagines doing together with others - the "move outside the monogamous form" requires a collective process to find another way.

\section{Polyamorous Temporalities}

According to Halberstam (2005), heteronormativity is not just about which bodies are expected to desire each other but also the view of life as a progressive story in which couple bonding and reproduction is the goal and meaning. Not participating in that story or changing its chronology, for example, by not having children - or having them at the wrong age-or not being part of a coupled relationship is seen as a sign of immaturity. Lo reflects on this:

You're supposed to find a partner, and then you will live happily ever after. That person is supposed to be closest and fill all your needs, and if not, there is something wrong with the relationship. And you have to have children and dinners with other couples. You have to eat breakfast together and go on holidays and love weekends together, and everything will be with that person. Then, finally, you are home and have found your soul mate. But... you might already have soul mates or you might want several close relationships in your life.

People living a queer life are not only seen as incomprehensible, but also perceived as having something wrong with them due to their relationships (Halberstam 2005). Lo clearly describes each phase of life and what is expected during each. But according to Lo, this is too limited. As she says, there may already be soul mates in your life, and there may be other relationships that need to be prioritized, thus describing collective aspects of living outside of the expected phases.

A person who does not follow the expected chronology of age and life stage, such as marriage, risks not being understood, thus ending up in a queer time beyond heteronormativity. Denise reflects on this:

I have been involved in several situations where my polyamorous friends have talked about their relationship and that they are getting married. And then people say, "Well, you're getting married, so you're not going to have an open relationship anymore?" [They answer] "Well, we'll have an open relationship, even though we're married." And then people get like... they short-circuit, just "huh?!" They don't understand anything.

Halberstam (2005) argues that falling in line with our expected life phase helps maintain a respectable and age-bound heterosexuality. By acting in a way that is not accepted in relation to one's life stage, one risks being perceived as immature and unable to solve the heteronormative life puzzle. A marriage is a clear marker of respectability, responsibility, and duration and is heavily based in Christian 
traditions and heteronormative culture where the decision to marry means that the couple has chosen each other and aims at a deeper step into the coupled relationship. As Denise explains, this then means that continuing with an open relationship in married life becomes a violation to respectable heterosexuality.

Christina, Carl, and Charlie live in a polyamorous relationship in the same household. Christina and Carl are in their 60s and are married. Charlie, who is in their thirties, is their partner. During the interview, normative expectations linked to different stages of life, age, and parenting are completely turned around:

Christina: But sometimes I've thought like... yes, no, but is Charlie my son? No, absolutely not. I do not feel like you were my son, but if you were to have children, I would probably feel like they were my grandchildren. Though you're not my son. [...]

Charlie: But I do not decide like that. I have feelings like, sometimes I feel like Christina and Carl are my children, sometimes I feel they are my partners, and sometimes I feel they are my dad and mom. I feel... I always have the feelings in different ways.

Queer subcultures, Halberstam (2005) notes, "produce alternative temporalities by allowing their participants to believe that their futures can be imagined according to logics that lie outside of those paradigmatic markers of life experience-namely, birth, marriage, reproduction, and death" (p. 2). In the conversation above, the (heterosexual) normative fixed roles as children, partners, and parents are dissolved, and instead, the informants describe how they seize the different positions at different times and in different emotion modes. Here, being a parent is understood as a position of responsibility, care, and solicitude rather than biological factors and inheritance. Adopting the role of a child may offer the possibility to feel small, pampered and loved unconditionally. Christina says, "Sometimes I can feel, 'Oh, you're the child I never had' and in the next moment, 'but you make me horny', by which she creates permission to try out the different positions of what it means to be both lovers and parents as well as letting the feelings that follow be allowed to exist. The conversation also shows how normative life phase expectations can be transformed into queer temporalities when Christina says, "I do not feel like you were my son, but if you were to have children, I would probably feel like they were my grandchildren." Queer temporalities have "the potentiality of a life unscripted by the conventions of family, inheritance, and child rearing" (p. 2), an example of which is with Christina's wish to be a grandparent to her lovers' children.

\section{Silence and Coming Out}

Not all stories are about expanding opportunities - there are also important themes of silence and issues of coming out and being met with ignorance or prejudice. The degree of openness is something that polyamorous people inevitably have to reflect upon in their everyday life. All informants describe difficulties to live as poly in a society where monogamy is norm. In the interviews, it became obvious that coupled relations are praised while other relationship constellations imply different 
sanctions. Several informants describe how people in their surroundings find it difficult to understand their way of forming relationships and they are often faced with scepticism and negative comments. Sara thinks it is difficult to talk about problems that occur in her relationships because it is always explained away as a result of her non-monogamy:

When you have a hard time in your relationship or in one of the relationships, I have received responses like "Yes, but it is clear that you are having a hard time because you live like that. It must be really complicated and really problematic and it is no wonder it creates conflicts." Or "It is clear that that sort of relationship cannot last forever." But no one would use it as an explanation to a failing monogamous relationship by saying "Yes, but it is because you agreed to monogamy."

Here, Sara describes how especially hard it is to not be able to articulate issues in polyamorous relationships due to being met with the reaction that these types of relationships are not sustainable. Based on interviews, Klesse's (2007a, b) study found that one of the dominant narratives surrounding polyamory is the value placed on long-term relationships founded on honesty, love, understanding, and trust. Although previous studies show that stability, durability, and intimacy are important aspects of polyamorous relationships, like Sarah, several informants testify that the mainstream view reproduces non-monogamous relations as unstable, problematic, and occasional. Given that polyamory is associated with stigma, openness is associated with risks regarding social relationships and professional life (Conley et al. 2013). When Jasmine is asked if she has ever received positive responses from people around her based on how she lives, she answers, "No, I can't really say that I have... no, I don't think so." Instead, she thinks there is a constant clash with society's norms:

I think the hardest thing is that you are not taken seriously, either by society or by other people. That it's like, "Well, you're like that. You want to fuck around." (...) We are seen as unreliable, and I think that image needs to disappear. Because it's a bit sad to be seen as irresponsible and unreliable all the time. Because we are also serious. We also break. We also get heartache. We are also people.

Jasmine succinctly describes the ramifications of being out of line, of orienting her body in a different direction. Jasmine's polyamorous relationships are written off as "fucking around", and her emotions and feelings of love are not taken seriously. People living a queer life are not only seen as incomprehensible, but also they risk being perceived as immature or childish and incapable of having serious relationships (Halberstam 2005). Although Swedish society is characterized as liberal and open in relation to intimacy and sexuality, the majority of the informants feel forced to conceal their lifestyle in different situations and contexts. Calculating the risk of coming out and being seen as different and strange is repeatedly described. Love, for example, says, 
There are so many things I need to come out with, because everything I am and do, how I live and what I eat, I need to come out with all the time. So, I cannot do that. And I cannot explain, and I feel very, very weird most of the time. Yes. And I actually think that to be polyamorous, along with being non-binary, are the two norms I break that are the most difficult to be open with. I think I know how people would react, and that's why I'm not coming out. People would think I am weird and be questioning, and I would have to educate them. And they would criticize me.

Linn, who is bipolar, bisexual, polyamorous, and a BDSM practitioner describes how she has to come out of a "variety of wardrobes":

I am inside a lot of wardrobes which I have to come out of all the time. And all these wardrobes consist of groups that are stigmatized: To be bipolar is the freak-wardrobe. Practicing BDSM is taboo. To be bisexual means you are invisible in society. Non-monogamous persons are a group of people who can't decide how to live.

Halperin (1995, p. 30) writes, "To come out is precisely to expose oneself to a different set of dangers and constraints, to make oneself into a convenient screen onto which straight people can project all the fantasies they routinely entertain about gay people." Although Halperin refers to homosexuality's coming-out process, his description is relevant also to polyamorous persons. The preconceived opinions and fantasies about what a polyamorous lifestyle means are projected onto the individual, meaning that he or she needs to explain, deny, or elucidate what it means to him or her. This becomes clear in both Linn's and Love's interview extracts and is also something that informant David testifies about. His colleagues confronted him when they realized that he went out with more than one woman, and he felt he needed to tell them about his lifestyle:

They asked, "What are you doing?" and insisted that I cannot do that to my partner. I answered, "I can, because she knows all of them... she knows what I'm doing and she accepts it." and I had to have a shorter talk about it. And then came the questions: "How is it, and how are you doing it?" But I felt it became very sexist. Especially from guys my age or over. At first, they said, "Wow, that's wonderful, you can sleep with however many you want to!" Then, it was just as if the insight came that your partner also may be with however many she wants. Then they said, "You cannot have it like that. You cannot let her do that."

The risk of being given the reputation of being unfaithful and unreliable when it comes to relationships is recurrent in the informants' stories. David tells how he receives what he sees as sexist comments concerning how lucky he is to be sleeping with many women but that he should not "let her" do the same. Silence is strategically used to avoid being accused of unreliability or becoming incomprehensible. 


\section{Being Stopped-Interactions with Professionals}

While silence is used to avoid negative reactions, some interviewees tell of situations when they chose to come out or in other ways negotiate talking about their polyamorous practices. Ahmed (2006) describes how bodies that do not follow the expected lines are stopped or blocked. These blockages can lead to what Ahmed calls "stress points": "When such lines block rather than enable action they become points that accumulate stress, or stress points" (p. 160). One informant relates how her polyamorous female friends avoid getting tested for sexually transmitted infections because "they feel like sluts because they're always questioned when they seek healthcare." How we live as sexual beings is closely connected to shame and respectability (Skeggs 1997). Halberstam (2005) argues that the concept of queer time can "make clear how respectability, and notions of the normal on which it depends, may be upheld by a middle-class logic of reproductive temporality" (p. 4).

Several informants reflect on the negative response and lack of knowledge about non-monogamy among persons working in the caring professions. Jenny tells about her experiences of therapy:

I have seen several therapists, and then it has often been that I talk about my life and relationships, and I feel that there is a very, very little competence about non-monogamous relations. [...] I have been with two psychologists who insisted that there had to be something wrong - either it was my childhood, or that I have problems in relation to my mother, or that it would come from sexual exploitation as a child. It was like that. I felt it instantly. This is what they want to force out of me.

To continuously be stopped and questioned often leads to reactions such as frustration, shame, and even trauma (Ahmed 2006). Jenny articulates the violent way that she experienced her therapists wanting her to force out a cause that would explain the non-monogamy: "I felt a bit like a freak in relation to the normative world," she says in another part of the interview.

Lack of knowledge and competence about polyamory exists within different social institutions, not only healthcare but also authorities such as the Migration Board. As mentioned, Christina, Carl, and Charlie live in a polyamorous relationship, and Christina and Carl are married. Charlie wants to apply for an extended residence permit and states the relationship with Carl and Christina as a reason for extending the residence permit. When contacting the Migration Board in regards to the issue, they received the following response (which is used and translated with the permission of the informants):

Unfortunately, there is no possibility for you to book a time to talk about this with us. If you have questions, please send them via email or phone. We have no expert on this particular topic because we do not accept a polyamorous relationship. In Sweden, you are only allowed to have one partner. If there is an extension application where he applies to live in Sweden because of your relationship, you can have a dialogue with your migrations officer, but it is important to note that we do not accept these types of relationships. 
The Migration Board's response contains several inaccuracies. There is no law in Sweden stipulating that we can only live with one partner. The law states that we can marry only one person; however, polyamory and polygamy are two different things. Polyamorous relationships are not forbidden in Swedish society. The blockage in this case has potentially serious ramifications for Charlie's possibility of receiving an extended residence permit. By disregarding the fact that the relationship between Charlie, Christina, and Carl is not a marital one, but one of cohabiting, the representatives of the Migration Board can use the Marriage Act in their response to try to discredit the application and, in the words of Ahmed, stop Charlie from being able to continue practicing polyamory. Speaking about polyamory in different contexts, the interviewees tell of how they and people in their surroundings are stopped in different ways. Articulating non-monogamy goes beyond mono-normativity and expectations of life phases, but as evident in the interviewees' statements regarding therapy and the Migration Board, non-monogamy also goes beyond the logics of psychology and bureaucracy.

\section{Conclusion}

Our existence is constantly shaped by the environment in which we live. Swedish society is characterized by a unique ideological tradition of gender equality and respect for an individual's choice to form intimate relationships. Nevertheless, the majority of Swedes live in heterosexual monogamous relationships, and choosing lines other than the heteronormative line still implies risks. The majority of the informants feel forced to conceal their relationship constellations in several situations and contexts. Moving beyond the path and orienting one's body in another direction risks being hindered by both ignorance and prejudice, but it can also be important for opening up new possibilities both in relation to oneself and to others. In this article, we have investigated turning points that can be seen in the informants' stories, in the queer temporalities that are created and in orientations toward other polyamorous or queer bodies that offer spaces for collective change. The majority of the informants tell about a certain occasion in life when they first experienced polyamory, which they also refer to as a turning point. Learning of others who have chosen a polyamorous orientation has great importance in the informants' lives. This opens up the possibility to choose a line of polyamory or non-monogamy when a turning point occurs, such as a divorce or meeting someone and falling in love. Sometimes the informants' lives are completely rearranged and disorganized, but at the same time, they tell of the possibilities of living outside of protocols and templates.

In the theoretical language of Ahmed (2006), living a life within monogamous boundaries would be considered being "in line". The interviewees tell of heteronormative templates and norms that act as guidelines in life and when violated, certain sanctions occur. Both age and gender become important components in the performance of achieving respectability, when Linn, for example, describes how she has violated the norms for what is correct behavior for a woman of her age. Temporality as a way of organizing one's life in normative or non-normative ways is also at play 
in determining who is serious and has a mature and appropriate way of handling relationships (Halberstam 2005). In this way, alternative or queer temporalities are produced, which for some of the informants means their experiences are invalidated and they are being perceived as immature or unreliable. The risks of being stopped and hopes of re-orientation are crucial elements for the possibilities of choosing a lifestyle that goes beyond heteronormative expectations of monogamy. Interactions with healthcare professionals seldom offer a relief from this. Instead, the informants' stories of these encounters can be interpreted as instances of being stopped and blocked, resulting in stress and shame in the worst cases.

Our findings show how polyamory can offer alternative paths of life and relationships to other queer and polyamorous bodies in a society where the nuclear family and coupledom still are privileged and represent strong ideas of maturity, seriousness, and adulthood. It is important to point out that people who are polyamorous do not constitute a homogeneous group but have a diversity of experiences. Research on polyamory that further develops intersectional approaches is necessary to reflect the diversity of experiences the group holds and can generate understandings of how systems of power intersect and coproduce one another resulting in unequal material realities.

Funding This study was funded by the Centre for Sexology and Sexuality studies at Malmö University.

\section{Compliance with Ethical Standards}

Conflict of interest The authors declare that they have no conflict of interest.

Ethical Approval All procedures performed in studies involving human participants were in accordance with the ethical standards of the institutional and/or national research committee and with the 1964 Helsinki Declaration and its later amendments or comparable ethical standards.

Informed Consent Informed consent was obtained from all individual participants included in the study.

Open Access This article is distributed under the terms of the Creative Commons Attribution 4.0 International License (http://creativecommons.org/licenses/by/4.0/), which permits unrestricted use, distribution, and reproduction in any medium, provided you give appropriate credit to the original author(s) and the source, provide a link to the Creative Commons license, and indicate if changes were made.

\section{References}

Ahmed, S. (2006). Queer phenomenology. Orientations, objects, others. Durham: Duke University Press.

Anapol, D. (2010). Polyamory in the 21st century. Maryland: Rowman and Littlefield Publishers.

Balzarini, R. N., Dharma, C., Kohut, T., Holmes, B. M., Campbell, L., Lehmiller, J. J., et al. (2018). Demographic comparison of American individuals in polyamorous and monogamous relationships. The Journal of Sex Research. https://doi.org/10.1080/00224499.2018.1474333.

Barker, M. (2005). This is my partner, and this is my... partner's partner: Constructing a polyamorous identity in a monogamous world. Journal of Constructivist Psychology, 18(1), 75-88.

Barker, M., \& Langdridge, D. (Eds.). (2010). Understanding non-monogamies. New York: Routledge. 
Bauer, R. (2010). Non-monogamy in queer BDSM communities: Putting the sex back into alternative relationship practices and discourse. In M. Barker \& D. Langdridge (Eds.), Understanding nonmonogamies (pp. 142-153). New York: Routledge.

Bertilsdotter Rosqvist, H. (2007). Lagom lika, lagom olika: En diskussion om makt, retorik och biteoretiska/sexuella subjektiviteter. Ph.D. thesis, Umeå University, Umeå.

Braun, V., \& Clarke, V. (2006). Using thematic analysis in psychology. Qualitative Research in Psychology, 3(2), 77-101.

Bremer, S. (2011). Kroppslinjer. Kön, transexualism och kropp i berättelser om könskorrigering. Gothenburg: Makadam.

Carlström, C., \& Andersson, C. (2019). The queer spaces of BDSM and non-monogamy. Journal of Positive Sexuality, 5(1), 14-19.

Codex. (2012). Research ethical principles in the arts and social sciences). Stockholm: Swedish Research Council.

Conley, T., Moors, A., Matsick, J., \& Ziegler, A. (2013). The fewer the merrier? Assessing stigma surrounding consensually non-monogamous romantic relationships. Analyses of Social Issues and Public Policy, 13, 1-30.

Deri, J. (2015). Love's refraction. Jealousy and compersion in queer polyamorous relationships. Toronto: University of Toronto Press.

Dinshaw, C., Edelman, L., Ferguson, R. A., Freccero, C., Freeman, E., Halberstam, J., et al. (2007). Theorizing queer temporalities: A roundtable discussion. GLQ: A Journal of Lesbian and Gay Studies, 13(2), 177-195.

Gustavson, M. (2006). Blandade känslor: bisexuella kvinnors praktik \& politik. Ph.D. thesis, Linköpings university, Linköping.

Halberstam, J. (2005). In a queer time and place: Transgender bodies, subcultural lives. New York: New York University Press.

Halperin, D. M. (1995). Saint foucault. Towards a gay hagiography. Oxford: Oxford University Press.

Haupert, M. L., Gesselman, A. N., Moors, A. C., Fisher, H. E., \& Garcia, J. R. (2017). Prevalence of experiences with consensual nonmonogamous relationships: Findings from two national samples of single Americans. Journal of Sex and Marital Therapy, 43(5), 424-440.

Hutzler, K. T., Giuliano, T. A., Herselman, J. R., \& Johnson, S. M. (2016). Three's a crowd: Public awareness and (mis)perceptions of polyamory. Psychology \& Sexuality, 7(2), 69-87.

Klesse, C. (2006). Polyamory and its "Others": Contesting the terms of non-monogamy. Sexualities, 9(5), 565-583.

Klesse, C. (2007a). "How to be a happy homosexual?!" Non-monogamy and governmentality in the relationship manuals for gay men in the 1980s and 1990s. Sociological Review, 55(3), 571-591.

Klesse, C. (2007b). The spectre of promiscuity: Gay male and bisexual non-monogamies and polyamories. Burlington: Ashgate.

Klesse, C. (2018). Theorizing multi-partner relationships and sexualities-Recent work on nonmonogamy and polyamory. Sexualities, 21(7), 1109-1124.

Kolesar, A. (2011). Identity development at the intersections of bisexuality and polyamory. In American Psychological Association 2011, Convention Presentation.

Matsick, J. L., Conley, T. D., Ziegler, A., Moors, A. C., \& Rubin, J. D. (2014). Love and sex: Polyamorous relationships are perceived more favourably than swinging and open relationships. Psychology \& Sexuality, 5(4), 339-348.

Pain, E. (2019). Queer polyfamily performativity: Family practices and adaptive strategies among LGBTQ + polyamorists. Journal of GLBT Family Studies. Published online: 22 Apr 2019.

Pallotta-Chiarolli, M. (2010). to pass, border or pollute-Polyfamilies Go To School. In M. Barker \& D. Langdridge (Eds.), Understanding non-monogamies (pp. 182-187). New York: Routledge.

Ritchie, A., \& Barker, M. (2006). There aren't words for what we feel so we have to make them up: Constructing polyamorous languages in a culture of compulsory monogamy. Sexualities, 9(5), 584-601.

Rosenberg, T. (2002). Queerfeministisk agenda. Stockholm: Atlas.

Rubin, J. D., Moors, A. C., Matsick, J. L., Ziegler, A., \& Conley, T. D. (2014). On the margins: Considering diversity among consensually non-monogamous relationships. [Special Issue on Polyamory]. Journal für Psychologie, 22(1), 19-37.

Séguin, L. J. (2017). The good, the bad, and the ugly: Lay attitudes and perceptions of polyamory. Sexualities. https://doi.org/10.1177/1363460717713382. 
Sheff, E. (2005). Polyamorous relationships: Exploring community, gender, family and sexuality. Ann Arbor: ProQuest Information and Learning Company.

Sheff, E., \& Hammers, C. (2011). The privilege of perversities: Race, class and education among polyamorists and kinksters. Psychology \& Sexuality, 2(3), 198-223.

Skeggs, B. (1997). Formations of class and gender: Becoming respectable. London: Sage.

Tweedy, A. E. (2011). Polyamory as a sexual orientation. University of Cincinnati Law Review, 79(4), 1461-1515.

Wilkinson, E. (2010). What's queer about non-monogamy now? In M. Barker \& D. Langdridge (Eds.), Understanding non-monogamies (pp. 243-254). New York: Routledge.

Williams, D. J., \& Prior, E. E. (2015). Commentary: Contemporary polyamory: A call for awareness and sensitivity in social work. Social Work, 60(3), 268-270.

Wosick-Correa, K. (2010). Agreements, rules and agentic fidelity in polyamorous relationships. Psychology \& Sexuality, 1(1), 44-61.

Publisher's Note Springer Nature remains neutral with regard to jurisdictional claims in published maps and institutional affiliations. 